\title{
An Unambiguous Tracking Technique for Cosine-Phased BOC Signals with Low Complexity
}

\author{
Tian LI, Zuping TANG, Jiaolong WEI, Zhihui ZHOU, Boyi WANG
}

School of Electronic Information and Communications, Huazhong University of Science and Technology, China

litianpiece@163.com, tang_zuping@hust.edu.cn,jwei@mail.hust.edu.cn, zhih_zhou@163.com, boyiwang05@gmail.com

Submitted April 25, 2018 / Accepted September 4, 2018

\begin{abstract}
A low-complexity unambiguous tracking method for cosine-phased binary offset carrier $(B O C$ C) signals is proposed in this paper. The proposed method directly constructs a code discriminator function by multiplying two correlation functions. One local reference signal is a specifically designed auxiliary signal whose cross-correlation function with the BOCc signal is an unambiguous $S$-curve. The other reference signal is a replica BOCc signal whose correlation function with the BOCc signal is used as a "cover" to maintain the slope of the discriminant function as much as possible and to make the final discriminant function noncoherent. The proposed discriminator function has only a single main lock point and can make tracking reliable and unambiguous. In contrast to the traditional unambiguous early-minus-late methods, the proposed method needs only the prompt branch correlator outputs, and the correlation process of the BOCc signal with input signals is the same as that of the carrier loop process. As a result, the proposed method reduces the number of correlators by at least three-quarters. The theoretical analysis and simulation results show that the proposed method has higher code tracking accuracy, lower tracking threshold and better anti-multipath performance than those of PUDLL, SF and SPAR. In conclusion, the proposed method completely eliminates tracking ambiguity, significantly improves tracking performance and reduces implementation complexity.
\end{abstract}

\section{Keywords}

BOC, unambiguous tracking, local auxiliary signal, low complexity, GNSS

\section{Introduction}

As an increasing number of navigation signals join the global navigation satellite system (GNSS), the compatibility and interoperability of signals have become a focus of signal design. Binary offset carrier (BOC) signals and their derivative signals can be separated from each other in frequency by the square waveform subcarrier, which can make full use of the navigation frequency band and improve the compatibility. In addition, BOC signals have better code tracking accuracy and anti-multipath performance than those of BPSK signals with the same code rate [1]. In this paper, a sine or cosine BOC signal is defined as $\operatorname{BOCs}(m, n)$ or $\operatorname{BOCc}(m, n)$, respectively, where $m$ denotes that the frequency of the subcarrier is $m \times 1.023 \mathrm{MHz}$ and $n$ denotes that the code rate is $n \times 1.023 \mathrm{Mcps}$. BOC signals are widely used in GNSS: the Galileo used BOCc $(10,5)$ at E6 for the public restricted service $(\mathrm{PRS})$ and $\operatorname{BOCc}(15,2.5)$ at $\mathrm{E} 1$ for the PRS [2]. However, the cost of these potential performance improvements is a decrease in tracking reliability [3] due to the multiple side peaks in the autocorrelation function (ACF) of BOC signals. Each of the side peaks is a false lock point that makes traditional tracking ambiguous. GNSSs perform the positioning function based on timing information. Thus, a timing offset due to a tracking error could result in a serious positioning error [4]. Therefore, eliminating ambiguity is the premise and focus of BOC signal processing [5].

Most ambiguity elimination technique are based on the pseudo-correlation function method. The core idea is to construct an unambiguous correlation function with specifically designed local auxiliary signals. Then, the discriminant function is obtained by means of early minus late. Since there are more side peaks and turning points in the ACF of BOCc signals than BOCs signals, which makes it more difficult to eliminate the ambiguity problem of BOCc signals. As a result, the performance of some methods is significantly worse when applied to BOCc signals than BOCs signals, such as the symmetrical pulse ambiguity removing (SPAR) technique [6], the method proposed by Shen Feng (SF) in [7], and PUDLL [8], which uses two local step-shaped waveforms. There are also some other methods that have good performance but are suitable for only BOCs signals, such as [1] and [9-11]. Compared with all the methods mentioned before, [10] and [11], which are previous works by our team, have obvious advantages in tracking accuracy and anti-multipath performance. However, they are suitable for only BOCs signals, and implementing the receiver costs a considerable amount. In this paper, we proposed an unambiguous tracking method specifically for $\operatorname{BOCc}(\mathrm{m}, \mathrm{n})$ signals. In order to remove the ambiguity threat, the proposed method construct an unambiguous discrimina- 
tor function by multiplying two correlation functions. There are three differences between the proposed method and those of [10] and [11]. Firstly, the proposed method can solve the ambiguity problem of cosine phased BOC signal, while both [10] and [11] are only applicable for sine phased BOC signals. Secondly, the proposed method directly generates the unambiguous discriminator function instead of generating an unambiguous correlation function first and then realizing the unambiguous discriminator function by early minus late. Finally, the new tracking loop structure reduces the number of correlators by at least three quarters than those in [10] and [11]. As a result, compared with all the other unambiguous tracking methods for BOCc signals, the proposed method has obvious advantages in complexity. The theoretical analysis and simulation results show that the theoretical analysis is correlect and the proposed method has better tracking accuracy and lower threshold compared with PUDLL, SF, SPAR. A comparison of the performance in a multipath environment is also offered in this paper: under medium multipath conditions, the proposed algorithm has the smallest multipath error envelope among all the abovementioned methods. In conclusion, the proposed method greatly enhances the performance of unambiguous BOCc signal tracking with the lowest complexity.

The rest of this paper is organized as follows. Section 2 discusses the ambiguity problem of BOCc signals and describes the local reference signal of the proposed algorithm. The unambiguous tracking loop is described and analyzed in Sec. 3. We provide simulation results and a performance comparison in Sec. 4, and the conclusions are presented in the final section.

\section{Signal Models and Local Auxiliary Signals}

The BOC signal received from one satellite is described in [12] as (1):

$$
\begin{aligned}
r(t)= & \sqrt{2 C} D\left(t-\tau_{0}\right) s_{\mathrm{BOC}}\left(t-\tau_{0}\right) \cos \left(2 \pi f_{\mathrm{IF}} t+\theta_{0}\right)+n_{0}(t) \\
= & \sqrt{2 C} D\left(t-\tau_{0}\right) c\left(t-\tau_{0}\right) p_{\mathrm{BOC}}\left(t-\tau_{0}\right) \cos \left(2 \pi f_{\mathrm{IF}} t+\theta_{0}\right) \\
& +n_{c}(t) \cos \left(2 \pi f_{\mathrm{IF}} t\right)-n_{s}(t) \sin \left(2 \pi f_{\mathrm{IF}} t\right)
\end{aligned}
$$

where $C$ is the power of the received signal, $D(t)$ is the navigation data message, $c(t)$ is the pseudo-random noise (PRN) code waveform, $\tau_{0}$ is the transmission delay, $f_{\mathrm{IF}}$ is the frequency of the carrier, $\theta_{0}$ is the initial phase of the carrier, and $n_{0}(t)$ is band-limited white noise. $n_{c}(t)$ and $n_{s}(t)$ are independent zero-mean Gaussian random processes that have the same double-sided power spectrum density $N_{0}$ [13]. $P_{\mathrm{BOC}}$ denotes the waveform of the chips and is expressed as $P_{\mathrm{BOC}}(t)=\operatorname{sign}\left[\cos \left(2 \pi f_{\mathrm{sc}} t\right)\right]$, where $\operatorname{sign}()$ is the signum function and $f_{\mathrm{sc}}$ is the frequency of the subcarrier. To facilitate the analysis, the baseband form of $r(t)$ can be expressed as:

$$
\begin{aligned}
r_{\text {base }}(t)= & \sqrt{2 C} D\left(t-\tau_{0}\right) c\left(t-\tau_{0}\right) p_{\text {BOC }}\left(t-\tau_{0}\right) \mathrm{e}^{\mathrm{j} \theta_{0}} \\
& +n_{c}(t)+\mathrm{j} n_{s}(t) .
\end{aligned}
$$

The expression of the ACF of the BOCc signal can be described as (3) using the method in [14], where $k=2 \mathrm{~m} / n$ is the order of the BOC signal [15], $l=0,1,2, \ldots, k-1, T_{\mathrm{C}}$ is the chip duration, and $T_{\mathrm{S}}=T_{\mathrm{C}} / k$ is the half-cycle of the subcarrier[16]. For comparison, Fig. 1 and Fig. 2 depict the ACFs and traditional non-coherent early-minus-late power (NELP) discrimination curves, respectively, of $\operatorname{BOCc}(10,5)$ and $\operatorname{BPSK}(1)$.

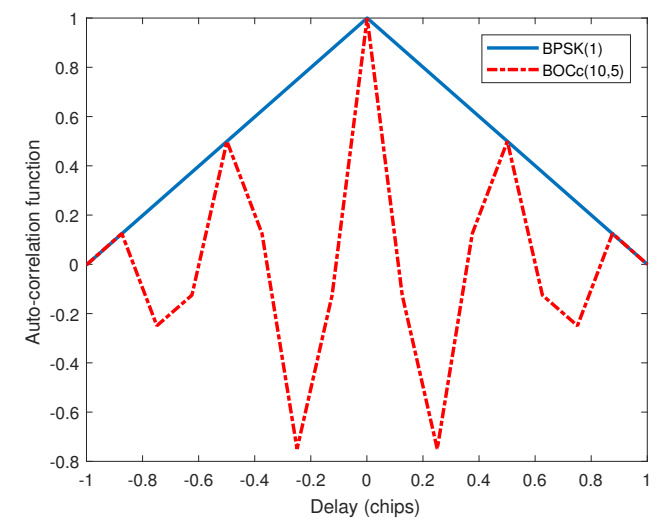

Fig. 1. The ACFs of BPSK(1) and $\mathrm{BOCc}(10,5)$.

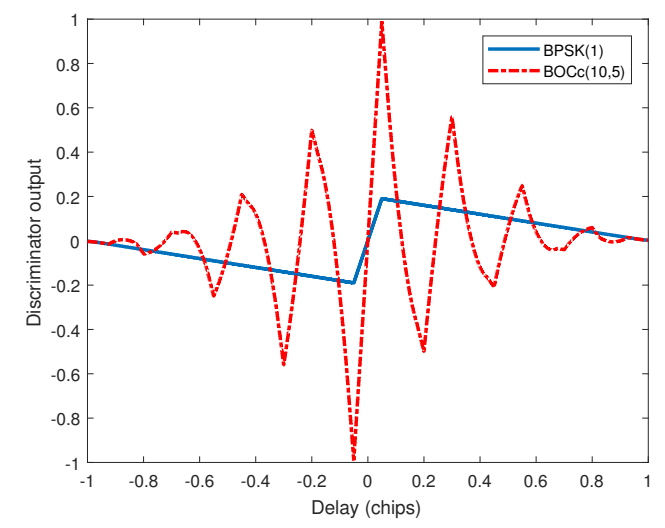

Fig. 2. The discriminator curves of $\operatorname{BPSK}(1)$ and $\operatorname{BOCc}(10,5)$.

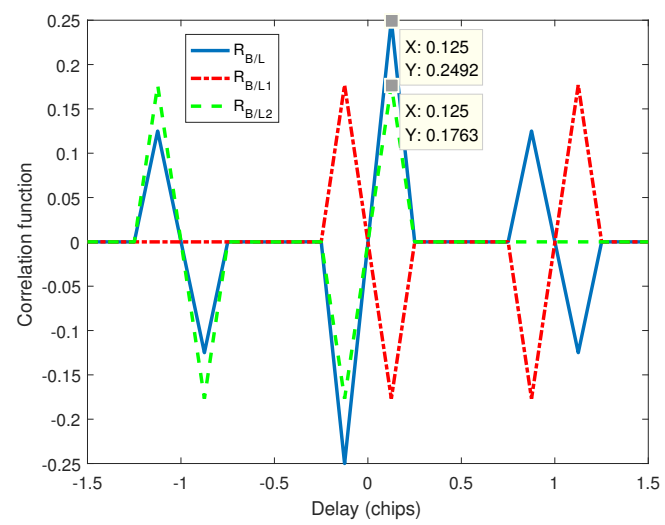

Fig. 3. The correlation functions of local auxiliary signals with $\operatorname{BOCc}(10,5)$. 


$$
R_{\mathrm{B}}(\tau)=\left\{\begin{array}{cr}
(-1)^{l}\left[\frac{(k-l)(2 l+1)+l}{k}-\frac{2 k-2 l+1}{T_{\mathrm{C}}}|\tau|\right] & l T_{\mathrm{S}} \leq|\tau|<\left(l+\frac{1}{2}\right) T_{\mathrm{S}} \\
(-1)^{l}\left[\frac{(k-l)(2 l+1)-3 l-2}{k}-\frac{2 k-2 l-3}{T_{\mathrm{C}}}|\tau|\right] & \left(l+\frac{1}{2}\right) T_{\mathrm{S}} \leq|\tau|<(l+1) T_{\mathrm{S}} \\
0 & |\tau| \geq T_{\mathrm{C}}
\end{array}\right.
$$

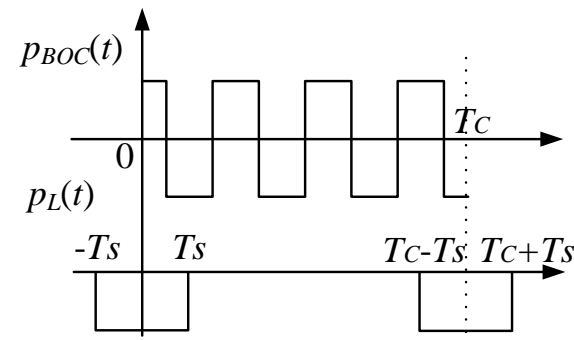

(a)

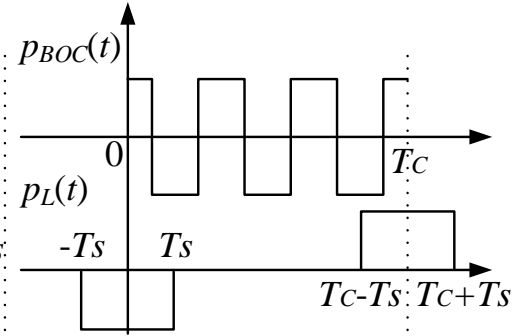

(b)

Fig. 4. Waveform of the proposed method. (a) Modulation order is odd, (b) modulation order is even

As shown in Fig. 1, compared with the triangle ACF of the BPSK signal, the main peak of the ACF of the BOC signal is narrower, which makes the BOC signal have better tracking accuracy and anti-multipath performance. However, BOCc has $2 k$ side peaks, and each side peak leads to a false lock point, as shown in Fig. 2, where the early-late spacing is 0.1 chips. It is catastrophic for the receiver to lock at any false lock points in the appeal. As a result, we must find a way to eliminate the ambiguity of BOC signal processing.

$P_{\mathrm{BOC}}(t)$ is a symmetric function with a period of $2 T_{\mathrm{S}}$ and meets the following indication:

$$
\int_{\tau}^{\tau+2 T_{\mathrm{S}}} P_{\mathrm{BOC}}(t) \mathrm{d} t=0 .
$$

Therefore, if the waveform of the local auxiliary signal is a rectangle with a width of $2 T_{\mathrm{S}}$, the number of side peaks in the CCF will decrease. Then, we can obtain two local reference signals:

$$
P_{\mathrm{L} 1}(t)=\left\{\begin{array}{cc}
\sqrt{k / 2} & -T_{\mathrm{S}}<t<T_{\mathrm{S}} \\
0 & \text { otherwise }
\end{array}\right.
$$

and

$$
P_{\mathrm{L} 2}(t)=\left\{\begin{array}{rr}
\sqrt{k / 2} & T_{\mathrm{C}}-T_{\mathrm{S}}<t<T_{\mathrm{C}}+T_{\mathrm{S}} \\
0 & \text { otherwise. }
\end{array}\right.
$$

The CCF $\left(R_{\mathrm{B} / \mathrm{L} 1}\right)$ of $P_{\mathrm{L} 1}$ with $\mathrm{BOCc}(10,5)$ and the $\mathrm{CCF}$ $\left(R_{\mathrm{B} / \mathrm{L} 2}\right)$ of $P_{\mathrm{L} 2}$ are shown in Fig. 3. When $t \approx 0, R_{\mathrm{B} / \mathrm{L} 2}$ is in the shape of an $\mathrm{S}$-curve, and $R_{\mathrm{B} / \mathrm{L} 1}$ is an inverse $\mathrm{S}$-curve. To further increase the slope of the S-curve when $t \approx 0, P_{\mathrm{L} 1}$ and $P_{\mathrm{L} 2}$ are combined to form the waveform of the local signal in (7), as shown in Fig. 4:

$$
P_{\mathrm{L}}(t)=\left\{\begin{array}{cc}
-\sqrt{k} / 2 & -T_{\mathrm{S}}<t<T_{\mathrm{S}} \\
(-1)^{k} \sqrt{k} / 2 & T_{\mathrm{C}}-T_{\mathrm{S}}<t<T_{\mathrm{C}}+T_{\mathrm{S}} \\
0 & \text { otherwise }
\end{array}\right.
$$

Then, the local auxiliary signal can be expressed as:

$$
s_{\mathrm{L}}(t)=\sum_{l} c_{l}(-1)^{k l} P_{\mathrm{L}}\left(t-l T_{\mathrm{C}}\right)
$$

where $c_{l}$ is the PRN code. Note that the power of the local auxiliary signal $s_{\mathrm{L}}(t)$ has been normalized. Since all the BOCc signals used in the GNSS are in even order, we consider only the case where $k$ is an even number for simplicity. When $k$ is an even number, we have $P_{\mathrm{L}}(t)=\sqrt{k} / 2\left[P_{T_{\mathrm{C}}}\left(t-T_{\mathrm{S}}\right)-P_{T_{\mathrm{C}}}\left(t+T_{\mathrm{S}}\right)\right]$, where $P_{T_{\mathrm{C}}}$ is the waveform of $\operatorname{BPSK}(n)$. Thus, $s_{\mathrm{L}}(t)$ is rewritten as:

$$
\begin{aligned}
s_{\mathrm{L}}(t) & =\frac{\sqrt{k}}{2} \sum_{l} c_{l}\left[P_{T_{\mathrm{C}}}\left(t-T_{\mathrm{S}}-l T_{\mathrm{C}}\right)-P_{T_{\mathrm{C}}}\left(t+T_{\mathrm{S}}-l T_{\mathrm{C}}\right)\right] \\
& =\sqrt{k} \frac{c_{l}\left(t-T_{\mathrm{S}}\right)-c_{l}\left(t+T_{\mathrm{S}}\right)}{2} .
\end{aligned}
$$

Equation (9) implies that the designed auxiliary signal can be generated by using the two different replicas of the PRN code, which is similar to the auxiliary signal in [10]. The correlation function $\left(R_{\mathrm{B} / \mathrm{L}}\right)$ of $S_{\mathrm{L}}$ with the BOCc signal is shown in Fig. 3 and (10).

$$
R_{\mathrm{B} / \mathrm{L}}(\tau)=\left\{\begin{array}{lc}
\frac{1}{\sqrt{k} T_{\mathrm{S}}} \tau & |\tau| \leq \frac{T_{\mathrm{S}}}{2} \\
\frac{\operatorname{sign}(\tau)}{\sqrt{k} T_{\mathrm{S}}}\left(T_{\mathrm{S}}-|\tau|\right) & \frac{T_{\mathrm{S}}}{2}<|\tau| \leq T_{\mathrm{S}} \\
\frac{1}{2 \sqrt{k} T_{\mathrm{S}}}\left(T_{\mathrm{C}}-|\tau|\right) & -\frac{T_{\mathrm{S}}}{2}<|\tau|-T_{\mathrm{C}} \leq-\frac{T_{\mathrm{S}}}{2} \\
\frac{1}{2 \sqrt{k} T_{\mathrm{S}}}\left(|\tau|-T_{\mathrm{C}}+T_{\mathrm{S}}\right) & -T_{\mathrm{S}}<|\tau|-T_{\mathrm{C}} \leq \frac{T_{\mathrm{S}}}{2} \\
\frac{1}{2 \sqrt{k} T_{\mathrm{S}}}\left(|\tau|-T_{\mathrm{C}}-T_{\mathrm{S}}\right) & \frac{T_{\mathrm{S}}}{2}<|\tau|-T_{\mathrm{C}} \leq T_{\mathrm{S}} \\
0 & \text { otherwise. }
\end{array}\right.
$$

As shown in Fig. 3, there is only one lock point in $R_{\mathrm{B} / \mathrm{L}}$ when $\tau=0$. The slope of $R_{\mathrm{B} / \mathrm{L}}$ is $\frac{0.2492 / 0.125}{0.1763 / 0.125}=1.414$ times better than those of $R_{\mathrm{B} / \mathrm{L} 1}$ and $R_{\mathrm{B} / \mathrm{L} 2}$. Note that when $\tau= \pm T_{\mathrm{c}}$, $R_{\mathrm{B} / \mathrm{L}}$ is an inverted S-curve, and the receiver will not lock at that point falsely, which means that $R_{\mathrm{B} / \mathrm{L}}$ is an unambiguous discriminant function for BOCc signals. 


$$
\begin{aligned}
R_{\text {un }}(\tau) & =\left[R_{\mathrm{B}}(\tau) \mathrm{e}^{\theta_{0}}\right]\left[R_{\mathrm{B} / \mathrm{L}}(\tau) \mathrm{e}^{\theta_{0}}\right]^{\prime} \\
& =R_{\mathrm{B}}(\tau) R_{\mathrm{B} / \mathrm{L}}(\tau) \\
& =\left\{\begin{array}{lc}
\frac{1}{\sqrt{k} T_{\mathrm{S}}} \tau\left(1-\frac{2 k+1}{T_{\mathrm{C}}}|\tau|\right) & |\tau| \leq \frac{T_{\mathrm{S}}}{2} \\
\frac{\operatorname{sign}(\tau)}{\sqrt{k} T_{\mathrm{S}}}\left(T_{\mathrm{S}}-|\tau|\right)\left(\frac{k-2}{k}-\frac{2 k-3}{T_{\mathrm{C}}}|\tau|\right) & \frac{T_{\mathrm{S}}}{2}<|\tau| \leq T_{\mathrm{S}} \\
\frac{(-1)^{k+1}}{2 k \sqrt{k} T_{\mathrm{S}}^{2}}\left(T_{\mathrm{C}}-|\tau|\right)^{2} & T_{\mathrm{C}}-\frac{T_{\mathrm{S}}}{2}<|\tau| \leq T_{\mathrm{C}} \\
\frac{(-1)^{k+1}}{2 k \sqrt{k} T_{\mathrm{S}}^{2}}\left(|\tau|-T_{\mathrm{C}}+T_{\mathrm{S}}\right)\left(3 T_{\mathrm{C}}-2 T_{\mathrm{S}}-3|\tau|\right) & -T_{\mathrm{S}}<|\tau|-T_{\mathrm{C}} \leq-\frac{T_{\mathrm{S}}}{2} \\
0 & \text { otherwise. }
\end{array}\right.
\end{aligned}
$$

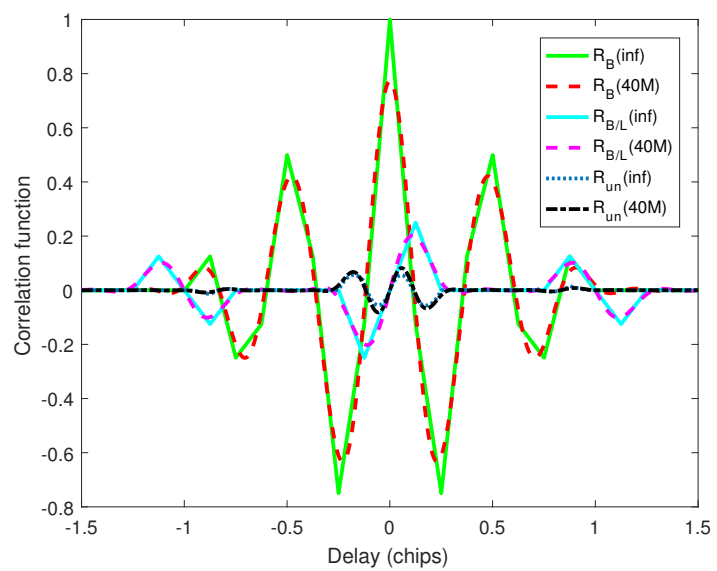

Fig. 5. The discriminator curves of $\operatorname{BOCc}(10,5)$.

However, it is worth noting that $R_{\mathrm{B} / \mathrm{L}}$ is a coherent discriminator function, which means that the performance of the discriminator is severely affected by the carrier residual phase. However, the carrier loop cannot completely eliminate the carrier phase, and the performance loss $(\cos (\Delta \theta))$ of the code loop caused by the residual carrier phase $(\Delta \theta)$ can not be ignored. To ensure that the code loop is relatively independent of the carrier loop, the receiver always selects non-coherent code loop discriminators. As a result, $R_{\mathrm{B}}$ is selected as a "hat" to maintain the slope of $R_{\mathrm{B} / \mathrm{L}}$ as much as possible and to make the final discriminator function non-coherent by multiplying it with $R_{\mathrm{B} / \mathrm{L}}$, as shown in (11), and the discriminator functions of BOCc $(10,5)$ are shown in Fig. 5, where inf denotes the correlation function obtained when the input signal is not filtered and 40M denotes the correlation function obtained when the input signal is filtered with a $40 \mathrm{MHz}$ bandwidth. As shown in Fig. 5, $R_{\text {un }}$ can be regarded as an unambiguous discriminator function under both filtered and unfiltered conditions. In conclusion, the method proposed in this paper is unambiguous.

\section{Tracking Scheme and Performance Analysis}

Figure 6 presents the proposed unambiguous tracking block diagram, where $I P_{1}$ and $Q P_{1}$ are the correlation output of the local BOC signal with the in-phase and quad-phase input signal, respectively, and $I P_{2}$ and $Q P_{2}$ are the correla- tion output of the local auxiliary signal with the in-phase and quad-phase input signal, respectively. Note that both $I P_{1}$ and $Q P_{1}$ are used in the code and carrier loops and that the carrier loop is indispensable to the tracking process, which means that the proposed method adds only $I P_{2}$ and $Q P_{2}$ to achieve code loop tracking. The four correlation outputs are:

$$
\begin{aligned}
& I P_{1}=\sqrt{2 C} R_{\mathrm{B}}(\Delta \tau) \operatorname{sinc}\left(\pi \Delta f T_{\mathrm{P}}\right) \cos (\Delta \theta)+n_{1}^{I}, \\
& Q P_{1}=\sqrt{2 C} R_{\mathrm{B}}(\Delta \tau) \operatorname{sinc}\left(\pi \Delta f T_{\mathrm{P}}\right) \sin (\Delta \theta)+n_{1}^{Q}, \\
& I P_{2}=\sqrt{2 C} R_{\mathrm{B} / \mathrm{L}}(\Delta \tau) \operatorname{sinc}\left(\pi \Delta f T_{\mathrm{P}}\right) \cos (\Delta \theta)+n_{2}^{I}, \\
& Q P_{2}=\sqrt{2 C} R_{\mathrm{B} / \mathrm{L}}(\Delta \tau) \operatorname{sinc}\left(\pi \Delta f T_{\mathrm{P}}\right) \sin (\Delta \theta)+n_{2}^{Q}
\end{aligned}
$$

where $\Delta f$ is the residual carrier frequency, $T_{\mathrm{P}}$ indicates the coherent integration time, and $\Delta \theta$ represents the residual carrier phase. When noise and $\Delta f$ are ignored, the non-coherent discriminator function $R_{\mathrm{un}}(t)$ is obtained from (13):

$$
\begin{aligned}
V(\Delta \tau)= & I P_{1} I P_{2}+Q P_{1} Q P_{2} \\
= & \sqrt{2 C} R_{\mathrm{B}}(\Delta \tau) \cos (\Delta \theta) \sqrt{2 C} R_{\mathrm{B} / \mathrm{L}}(\Delta \tau) \cos (\Delta \theta) \\
& +\sqrt{2 C} R_{\mathrm{B}}(\Delta \tau) \sin (\Delta \theta) \sqrt{2 C} R_{\mathrm{B} / \mathrm{L}}(\Delta \tau) \sin (\Delta \theta) \\
= & 2 C R_{\mathrm{un}}(\Delta \tau) \cos ^{2}(\Delta \theta)+2 C R_{\mathrm{un}}(\Delta \tau) \sin ^{2}(\Delta \theta) \\
= & 2 C R_{\mathrm{un}}(\Delta \tau) .
\end{aligned}
$$

When the tracking is in a steady state, $\Delta \tau \approx 0, \Delta f \approx 0$ and $\Delta \theta \approx 0$. The joint distribution of the correlator outputs has been well analysed in [17], as shown in (14):

$$
\left(I P_{1}, I P_{2}, Q E_{1}, Q L_{2}\right)^{\mathrm{T}} \sim N(\mu, \Sigma)
$$

with

$$
\mu=\sqrt{2 C} \operatorname{sinc}\left(\pi \Delta f T_{\mathrm{P}}\right)\left[\begin{array}{llll}
R_{\mathrm{B}}(0) & R_{\mathrm{B} / \mathrm{L}}(0) & 0 & 0
\end{array}\right]^{\mathrm{T}},
$$

and

$$
\Sigma=\frac{N_{0}}{T_{\mathrm{P}}}\left[\begin{array}{cccc}
1 & R_{\mathrm{B} / \mathrm{L}}(0) & 0 & 0 \\
R_{\mathrm{B} / \mathrm{L}}(0) & 1 & 0 & 0 \\
0 & 0 & 1 & R_{\mathrm{B} / \mathrm{L}}(0) \\
0 & 0 & R_{\mathrm{B} / \mathrm{L}}(0) & 1
\end{array}\right]
$$




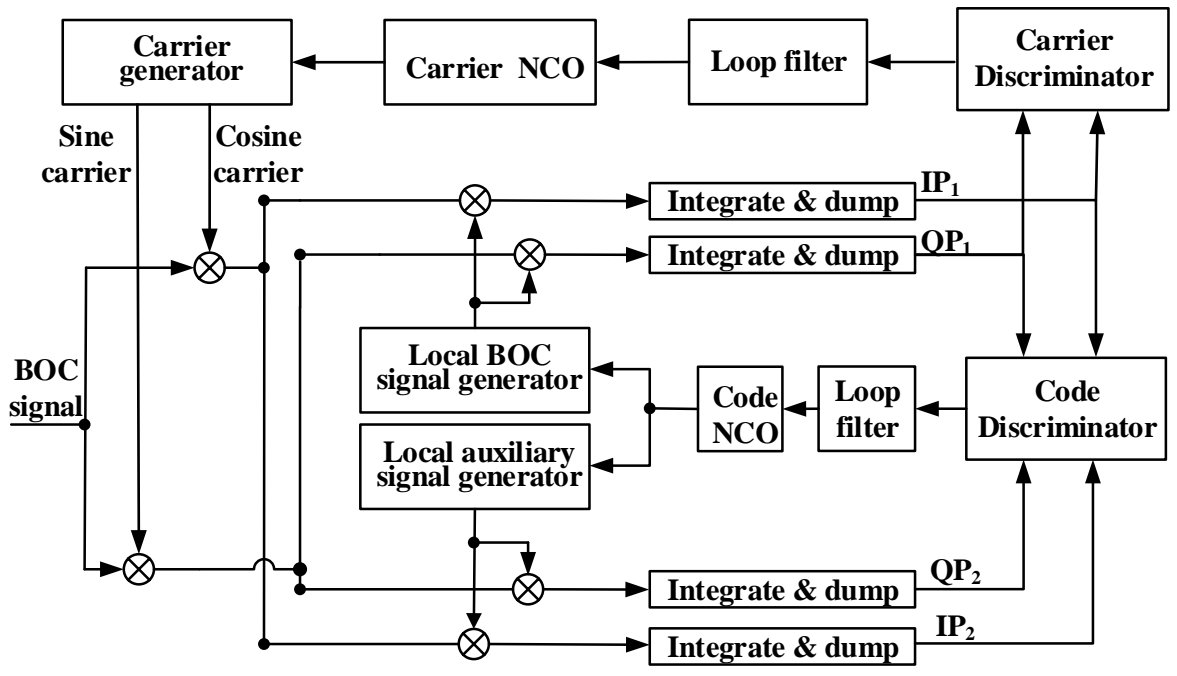

Fig. 6. Block diagram of the code and carrier loops.

The code tracking error variance has been thoroughly discussed in [16] and is given by (17):

$$
\sigma^{2}=\frac{2 \mathrm{~B}_{\mathrm{L}}\left(1-0.5 \mathrm{~B}_{\mathrm{L}} T_{\mathrm{P}}\right) T_{\mathrm{P}} \sigma_{V}^{2}}{K_{V}^{2}}
$$

where $B_{\mathrm{L}}$ is the single-sided code loop filter bandwidth, $\sigma$ is the discriminator output standard deviation, and $K_{V}$ is the discriminator gain. When considering the effect of a filter with transfer function $H(f)$, the correlation functions previously defined in (3) and (10) can be replaced by (18):

$$
\begin{aligned}
& \hat{R}_{\mathrm{B}}(\tau)=\mathrm{F}^{-1}\left[G_{\mathrm{B}}(f) H(f)\right], \\
& \hat{R}_{\mathrm{B} / \mathrm{L}}(\tau)=\mathrm{F}^{-1}\left[G_{\mathrm{B} / \mathrm{L}}(f) H(f)\right]
\end{aligned}
$$

where $G_{\mathrm{B}}(f)$ is the power spectral density of the BOCc signal, $G_{\mathrm{B} / \mathrm{L}}(f)$ represents the cross-power spectral density between BOCc and the local auxiliary signal, and $\mathrm{F}^{-1}$ denotes the inverse Fourier transform. The discriminator gain under bandwidth-limited conditions can be obtained by (19):

$$
\begin{aligned}
K_{V} & =\left.\frac{\mathrm{d} V}{\mathrm{~d} \Delta \tau}\right|_{\tau=0} \\
& =2 C \hat{R}_{\mathrm{B}}^{\prime}(0) \hat{R}_{\mathrm{B} / \mathrm{L}}(0)+2 C \hat{R}_{\mathrm{B}}(0) \hat{R}_{\mathrm{B} / \mathrm{L}}^{\prime}(0) \\
& =2 C \hat{R}_{\mathrm{B}}(0) \hat{R}_{\mathrm{B} / \mathrm{L}}^{\prime}(0) \\
& =4 \pi C \int_{-\infty}^{\infty} H(f) G_{\mathrm{B}}(f) \mathrm{d} f \int_{-\infty}^{\infty} f H(f) G_{\mathrm{B} / \mathrm{L}}(f) \mathrm{d} f .
\end{aligned}
$$

When the receiver is in a steady state, $V(t)$ is obtained by substituting (12) into (13):

$$
\begin{aligned}
V(0) & =\left(\sqrt{2 C} \hat{R}_{\mathrm{B}}(0)+n_{1}^{I}\right)\left(\sqrt{2 C} \hat{R}_{\mathrm{B} / \mathrm{L}}(0)+n_{2}^{I}\right)+n_{1}^{Q} n_{2}^{Q} \\
& =\sqrt{2 C} \hat{R}_{\mathrm{B}}(0) n_{2}^{I}+n_{1}^{I} n_{2}^{I}+n_{1}^{Q} n_{2}^{Q} \\
& =N_{1}+N_{2}+N_{3},
\end{aligned}
$$

where $N_{1}=\sqrt{2 C} \hat{R}_{\mathrm{B}}(0) n_{2}^{I}, N_{2}=n_{1}^{I} n_{2}^{I}$, and $N_{3}=n_{1}^{Q} n_{2}^{Q}$. As

$$
\mathrm{E}(V)=\mathrm{E}\left(N_{1}\right)+\mathrm{E}\left(N_{2}\right)+\mathrm{E}\left(N_{3}\right)=0
$$

we can obtain:

$$
\begin{aligned}
& \sigma_{V}^{2}=\mathrm{E}\left(V^{2}\right), \\
& \mathrm{E}\left(N_{1}^{2}\right)=\frac{2 C N_{0}}{T_{\mathrm{P}}} \hat{R}_{\mathrm{B}}^{2}(0), \\
& \mathrm{E}\left(N_{2}^{2}\right)=\mathrm{E}\left(N_{3}^{2}\right)=\left[1+2 \hat{R}_{\mathrm{B} / \mathrm{L}}^{2}(0)\right] \frac{N_{0}^{2}}{T_{\mathrm{P}}^{2}}=\frac{N_{0}^{2}}{T_{\mathrm{P}}^{2}}, \\
& \mathrm{E}\left(N_{1} N_{2}\right)=0, \\
& \mathrm{E}\left(N_{1} N_{3}\right)=0, \\
& \mathrm{E}\left(N_{2} N_{3}\right)=\frac{N_{0}^{2}}{T_{\mathrm{P}}^{2}} \hat{R}_{\mathrm{B} / \mathrm{L}}^{2}(0)=0 .
\end{aligned}
$$

Then,

$$
\begin{aligned}
\sigma_{V}^{2}= & \mathrm{E}\left(N_{1}^{2}\right)+\mathrm{E}\left(N_{2}^{2}\right)+\mathrm{E}\left(N_{3}^{2}\right) \\
& +2 \mathrm{E}\left(N_{1} N_{2}\right)+2 \mathrm{E}\left(N_{1} N_{3}\right)+2 \mathrm{E}\left(N_{2} N_{3}\right) \\
= & \frac{2 C N_{0}}{T_{\mathrm{P}}}\left[\hat{R}_{\mathrm{B}}^{2}(0)+\frac{1}{T_{\mathrm{P}} C / N_{0}}\right]
\end{aligned}
$$

By substituting (19) and (23) into (17), we can obtain the code tracking accuracy under additive Gaussian white noise, as shown in (24):

$$
\begin{aligned}
\sigma^{2} & =\frac{2 \mathrm{~B}_{\mathrm{L}}\left(1-0.5 \mathrm{~B}_{\mathrm{L}} T_{\mathrm{P}}\right) T_{\mathrm{P}} \sigma_{V}^{2}}{\left[4 \pi C \int_{-\infty}^{\infty} f H(f) G_{\mathrm{CS}}(f) \mathrm{d} f \int_{-\infty}^{\infty} f H(f) G_{\mathrm{B} / \mathrm{L}}(f) \mathrm{d} f\right]^{2}} \\
& =\frac{\mathrm{B}_{\mathrm{L}}\left(1-0.5 \mathrm{~B}_{\mathrm{L}} T_{\mathrm{P}}\right)\left[\hat{R}_{\mathrm{B}}^{2}(0)+\frac{1}{T_{\mathrm{P}} C / N_{0}}\right]}{4 \pi^{2} C / N_{0}\left[\int_{-\infty}^{\infty} H(f) G_{\mathrm{B}}(f) \mathrm{d} f \int_{-\infty}^{\infty} f H(f) G_{\mathrm{B} / \mathrm{L}}(f) \mathrm{d} f\right]^{2}} .
\end{aligned}
$$




\section{Simulation Results and Perfor- mance Comparison}

The main code tracking challenges in BOC signal processing are to avoid losing track of the signal (loss-of-lock situation), to operate well under noisy conditions, and to achieve high-accuracy code estimation under multipath channel conditions while preserving a reasonable complexity of the receiver [18]. With the exception of BPSK-Like(BL) [19], most unambiguous tracking algorithms have no specific requirement for the receiver front-end, loop filter, etc. With the same receiving parameters, only the number and type of correlators affect the complexity of different algorithms. The two local reference waveforms required in the proposed method are a BOCc signal and a pulse signal. Both waveforms can be classified as binary-level signals. For comparison, the correlator numbers of Bump-Jump (BJ) [20], BL, PUDLL, SF and SPAR are listed in Tab.1. Among all these methods, the proposed method requires the smallest number of binary-level correlators and no multilevel correlators. In other words, the proposed method has the lowest complexity and reduces the number of correlators by at least three-quarters.

Taking the actual use of BOCc signals into account, BOCc $(10,5)$ is selected as the simulation contrast signal. For comparison, the performances of BJ, BPSK-like, PUDLL, SF and SPAR are also provided. Figure 7 shows the code tracking performance of $\operatorname{BOCc}(10,5)$ with thermal noise. The code loop noise bandwidth $B_{\mathrm{L}}=1 \mathrm{~Hz}, T_{\mathrm{P}}=1 \mathrm{~ms}$, and the received bandwidth is $30.69 \mathrm{MHz}$ when the correlator interval is 0.1 chips. As shown in Fig. 7, compared with BPSKlike, PUDLL, SF and SPAR, the proposed method has the best performance. When the tracking error is 0.01 chips, the performance advantage of the proposed method over SPAR, PUDLL, SF, and BL is approximately $1 \mathrm{~dB}, 1.5 \mathrm{~dB}, 2 \mathrm{~dB}$, and $2.5 \mathrm{~dB}$, respectively. As the carrier-to-noise ratio decreases, the tracking accuracy advantage of the proposed algorithm increases.

\begin{tabular}{|c|c|c|}
\hline Algorithm & $\begin{array}{c}\text { Binary level } \\
\text { correlators }\end{array}$ & $\begin{array}{c}\text { Multi-level } \\
\text { correlators }\end{array}$ \\
\hline Proposed method & 2 & 0 \\
\hline BL & 8 & 0 \\
\hline BJ & 10 & 0 \\
\hline PUDLL & 0 & 8 \\
\hline SF & 0 & 8 \\
\hline SPAR & 8 & 0 \\
\hline
\end{tabular}

Tab. 1. Number of correlators.

\begin{tabular}{|c|c|}
\hline Algorithm & Tracking threshold $[\mathrm{dB}-\mathrm{Hz}]$ \\
\hline Proposed method & 28 \\
\hline BJ & 35 \\
\hline PUDLL & 31 \\
\hline SF & 33 \\
\hline SPAR & 33 \\
\hline
\end{tabular}

Tab. 2. Tracking threshold.

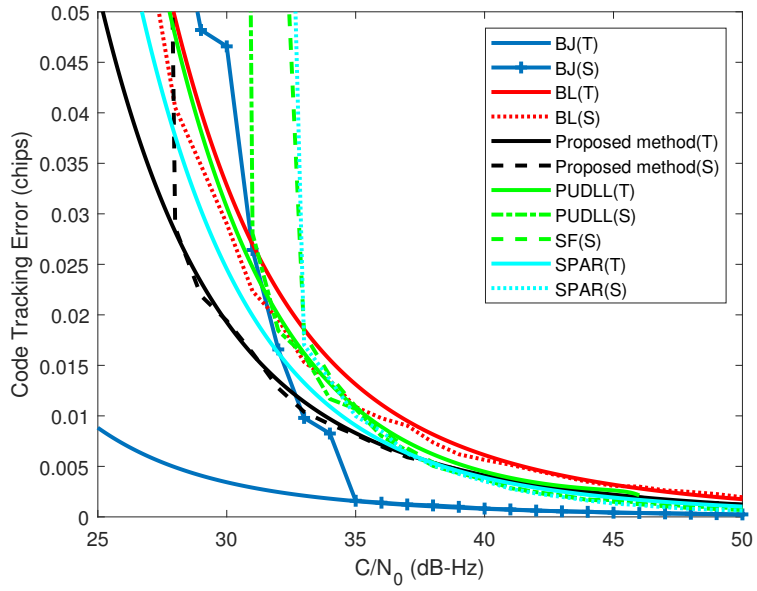

Fig. 7. Code tracking accuracy comparison for $\operatorname{BOCc}(10,5)$.

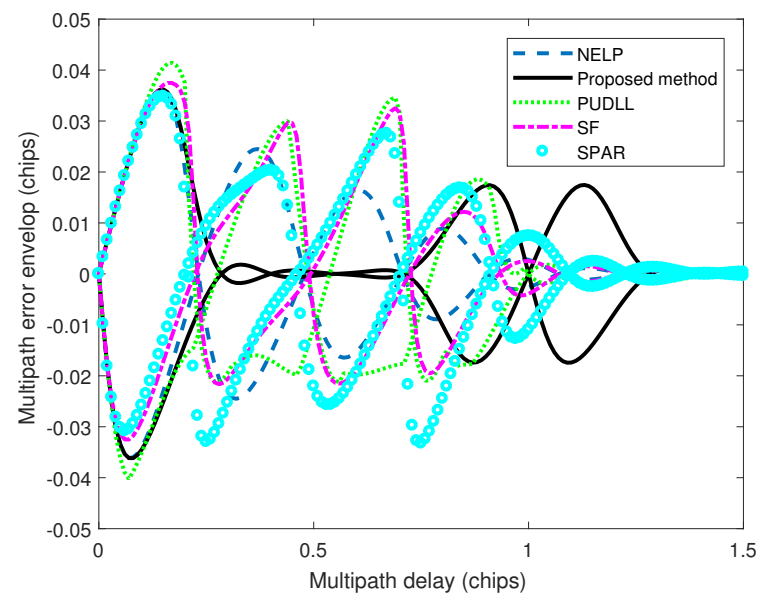

Fig. 8. Multipath error envelope for $\operatorname{BOCc}(10,5)$.

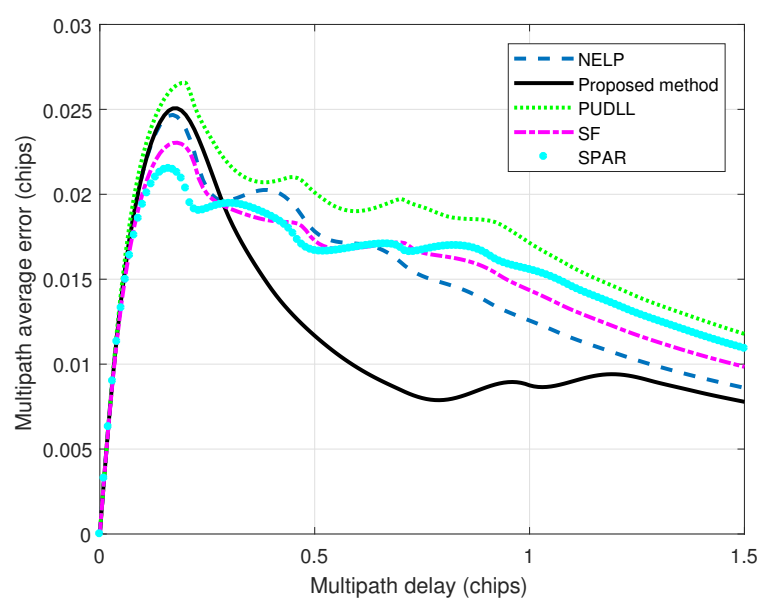

Fig. 9. Average multipath error for $\operatorname{BOCc}(10,5)$. 
BJ has the same tracking accuracy as NELP when false locking does not occur or when it rapidly jumps back to the main peak. However, as the environment worsens, the performance of $\mathrm{BJ}$ rapidly deteriorates, as shown in Fig. 7. BJ loses the lock when $\mathrm{C} / \mathrm{N} 0$ is less than $35 \mathrm{~dB}-\mathrm{Hz}$, whereas the proposed method loses the lock when $\mathrm{C} / \mathrm{N} 0$ is approximately $28 \mathrm{~dB}-\mathrm{Hz}$. The tracking thresholds of all the algorithms are listed in Tab. 2. The proposed method has the lowest code tracking threshold, which is $3 \mathrm{~dB}$ better than that of PUDLL, $5 \mathrm{~dB}$ better than those of SPAR and SF, and $7 \mathrm{~dB}$ better than that of BJ. That is, the proposed method has the best code tracking accuracy and robustness among the unambiguous tracking methods discussed above.

The anti-multipath performance of the proposed method is analysed and compared with those of SPAR, PUDLL, SF, BL, and NELP. The multipath model considered here is similar to that in [4]. The model includes one multipath signal with some amplitude attenuation relative to the direct signal. The phase difference between the multipath and direct signal is 0 or $\pi$. Figure. 8 and 9 show the multipath error envelope and the average multipath error, respectively, for $\operatorname{BOCc}(10,5)$ when the multipath-to-direct ratio is $-6 \mathrm{~dB}$. Note that the BPSK-like algorithm has poor performance under multipath conditions. To clearly compare the other algorithms, the performance of the BPSK-like algorithm is not depicted in the figure. In Fig. 8, when the multipath delay is between 0 and 0.25 chips or 0.75 and 1 chips, the performance of the proposed method is similar to those of PUDLL and SF. When the multipath delay is between 1 and 1.25 chips, the proposed method is sensitive to multipath signals. When the multipath delay is between 0.25 and 0.75 chips, the multipath signal has minimal influence on the proposed algorithm, whose anti-multipath performance is far better than those of all the other methods. As shown in Fig. 9, the anti-multipath performance advantage of the proposed method is clear.

\section{Conclusions}

In this paper, we proposed an unambiguous tracking technique with very low complexity. The method requires two local reproduced signals: a specifically designed binary auxiliary signal whose correlation function with BOCc signals is an unambiguous discriminator function and a BOCc signal whose correlation function with BOCc signals is the ACF. The second correlation is used as a "cover" to retain the discriminator slope as much as possible and to make the final discriminator function non-coherent by multiplying it with the first correlation function. Since only the output of the prompt branch is required, the proposed code tracking method reduces the number of correlators by at least one-half compared to that of traditional early-minus-late unambiguous tracking methods. In addition, since the correlation between the BOCc signal and the input signal is the same as the carrier tracking and the carrier tracking is indispensable to the receiver, the number of correlators actually required by the proposed code tracking method is less than one-quarter of those required by the other methods. The proposed method significantly reduces the complexity of the unambiguous tracking method.

Under thermal noise conditions, the code tracking accuracy advantage of the proposed method is clear: approximately $1 \mathrm{~dB}$ better than that of SPAR, $1.5 \mathrm{~dB}$ better than that of PUDLL and $2 \mathrm{~dB}$ better than that of SF. The code tracking threshold of the proposed method is $3 \mathrm{~dB}$ better than that of PUDLL, $5 \mathrm{~dB}$ better than those of SPAR and SF, and $7 \mathrm{~dB}$ better than that of BJ. In terms of multipath mitigation, the proposed method has the best average multipath error among all the algorithms. In summary, the proposed method achieves the best tracking accuracy and robustness while maintaining the lowest complexity.

\section{Acknowledgments}

This work is supported by the National Natural Science Foundation of China (NSFC), Grant 61401171.

\section{References}

[1] LI, T., WEI, J., TANG, Z., et al. An optimizing combined unambiguous correlation functions for boc signals tracking. In Proceedings of the International Technical Meeting of the Institute of Navigation. Monterey (USA), 2017, p. 388-400. ISSN: 2330-3646

[2] AVILA, J., HEIN, G., WALlNER, S., et al. The MBOC modulation: the final touch to the Galileo frequency and signal plan. Navigation, 2008, vol. 55, no. 1 , p. 15-28. DOI: $10.1002 / \mathrm{j} .2161-4296.2008 . t b 00415 . x$

[3] YAO, Z., GAO, Y., GAO, Y., et al. Generalized theory of BOC signal unambiguous tracking with two-dimensional loops. IEEE Transactions on Aerospace Electronic Systems, 2017, vol. 53, no. 6, p. 3056-3069. ISSN: 0018-9251. DOI: $10.1109 /$ TAES.2017.2726878

[4] CHAE, K., LEE, S.R., LIU, H. An unambiguous correlation function for generic sine-phased binary offset carrier signal tracking. Computers \& Electrical Engineering, 2016, vol. 49, no. C, p. 161-172. DOI: 10.1016/j.compeleceng.2015.06.023

[5] LI, T., WEI, J., TANG, Z., et al. An unambiguous acquisition technique for sine $\mathrm{BOC}(\mathrm{m}, \mathrm{n})$ signals. In Proceedings of the International Technical Meeting of the Institute of Navigation. Reston (USA), 2018, p. 13-20.

[6] QI, J., CHEN, J., LI, Z., ZHANG, D. Unambiguous BOC modulated signals synchronization technique. IEEE Communications Letters, 2012, vol. 16, no. 7, p. 986-989. ISSN: 1089-7798. DOI: 10.1109/LCOMM.2012.050112.112521

[7] SHEN, F., XU, G., CHEONG, J., FENG, H. Unambiguous acquisition and tracking technique for general BOC signals. Radioengineering, 2015, vol. 24, no. 3, p. 840-849. ISSN: 1210-2512, DOI: $10.13164 /$ re.2015.0840

[8] YAO, Z., et al. Pseudo-correlation function-based unambiguous tracking technique for sine-BOC signals. IEEE Transactions on Aerospace and Electronic Systems, 2010, vol. 46, no. 4, p. 1782-1796. DOI: 10.1109/TAES.2010.5595594 
[9] YAN, T., WEI, J., TANG, Z., et al. Unambiguous combined correlation functions for sine-BOC signal tracking. GPS Solutions, 2015, vol. 19, no. 4, p. 623-638. ISSN: 1080-5370. DOI: $10.1007 / \mathrm{s} 10291-014-0420-6$

[10] YAN, T., WEI, J., TANG, Z., et al. Unambiguous acquisition/tracking technique for high-order sine-phased binary offset carrier modulated signal. Wireless personal communications, 2015, vol. 84, no. 4, p. 2835-2857. DOI: $10.1007 / \mathrm{s} 11277-015-2769-4$

[11] LI, T., WEI, J., TANG, Z., et al. Unambiguous tracking technique based on combined correlation functions for sine boc signals. Journal of Navigation, 2018. DOI: 10.1017/S0373463318000498

[12] JULIEN, O., MACABIAU, C., CANNON, M., et al. ASPeCT: Unambiguous Sine-BOC(n,n) acquisition/tracking technique for navigation applications. IEEE Transactions on Aerospace and Electronic Systems, 2007, vol. 43, no. 1, p. 150-162. ISSN: 0018-9251. DOI: $10.1109 /$ taes.2007.357123

[13] YAN, K., ZIEDAN, N. I., ZHANG, H., et al. Weak GPS signal tracking using $\mathrm{fft}$ discriminator in open loop receiver. GPS Solutions, 2016, vol. 20, no. 2, p. 225-237. ISSN: 0018-9251. DOI: $10.1007 / \mathrm{s} 10291-014-0431-3$

[14] YAO, K., LU, M. Side-peaks cancellation analytic design framework with applications in BOC signals unambiguous processing. In Proceedings of the International Technical Meeting of the Institute of Navigation. San Diego (USA), 2011, p. 775-785.

[15] ZHANG, H., BA, X., CHEN, J., ZHOU, H. Unambiguous acquisition technique for $\mathrm{BOC}(\mathrm{m}, \mathrm{n})$ modulated signals. Hangkong Xuebao/Acta Aeronautica et Astronautica Sinica, 2017, vol. 38, no. 4, DOI: 10.7527/S1000-6893.2016.0194

[16] YAO, Z., LU, M., FENG, Z. Unambiguous sine-phased binary offset carrier modulated signal acquisition technique. IEEE Transactions on Wireless Communications, 2010, vol. 9, no. 2, p. 577-580. ISSN: 1536-1276. DOI: 10.1109/TWC.2010.02.091066

[17] KAO, T.L., JUANG, J.C. Weighted discriminators for GNSS BOC signal tracking. GPS Solutions, 2012, vol. 16, no. 3, p. 339-351. ISSN: 1080-5370. DOI: 10.1007/s10291-011-0235-7

[18] LOHAN, E.S., DIEGO, D.A.D., LOPEZ, J.A. Unambiguous techniques modernized GNSS signals: Surveying the solutions. IEEE Signal Processing Magazine, 2017, vol. 34, no. 5, p. 38-52. ISSN: 1053-5888. DOI: 10.1109/MSP.2017.2711778

[19] MARTIN, N., LEBLOND, V., GUILlotel, G., HEIRIES, V. BOC $(x, y)$ signal acquisition technique and performances. In Proceedings of the 16th International Technical Meeting of the Satellite Division of the Institute of Navigation (ION GPS/GNSS). Portland (USA), 2003, p. 188-198.

[20] FINE, P., WILSON, W. Tracking algorithm for GPS offset carrier signals. In Proceedings of the National Technical Meeting of The Institute of Navigation. San Diego (USA), 1999, p. 671-676.

\section{About the Authors...}

Tian LI was born in Liaoning, China, in 1990. He received his B.S. from Huazhong University of Science and Technology in 2013. He is currently working towards his Ph.D. at the School of Electronic Information and Communications, Huazhong University of Science and Technology, China. His research interests include next-generation GNSS signals and signal processing algorithms for GNSS receivers.

Zuping TANG (corresponding author) was born in Chongqing, China, in 1981. He received his B.S., M.Sc. and Ph.D. from Huazhong University of Science and Technology, Wuhan, China, in 2002, 2005 and 2009, respectively. He is currently an associate professor with the School of Electronic Information and Communications, Huazhong University of Science and Technology. His research interests include GNSS signal design theory, signal quality evaluation and GNSS receiver techniques.

Jiaolong WEI was born in Jiangxi, China, in 1965. He received his B.S., M.Sc. and Ph.D. from Huazhong University of Science and Technology, Wuhan, China, in 1986, 1990 and 2004, respectively. He is currently a professor, Ph.D. supervisor and vice-dean of the School of Electronic Information and Communications, Huazhong University of Science and Technology. His research interests include wireless communications and networks, intelligent computation and satellite navigation.

Zhihui ZHOU was born in Hunan, China, in 1988. He received his B.S. from Huazhong University of Science and Technology in 2011. He is currently working towards his $\mathrm{Ph} . \mathrm{D}$. at the School of Electronic Information and Communications, Huazhong University of Science and Technology, China. His research interests include next-generation GNSS signals and signal processing algorithms for GNSS receivers.

Boyi WANG was born in Hubei, China, in 1992. He received his B.S. from Wuhan University of Technology in 2014. He is currently working towards his Ph.D. in the School of Electronic Information and Communications, Huazhong University of Science and Technology, China. His research interests include next-generation GNSS signals and signal processing algorithms for GNSS receivers. 